AUFSÄTZE

Martin Rink Der kleine Krieg. Entwicklungen und Trends asymmetrischer Gewalt 1740 bis 1815

355

Klaus-Jürgen Bremm Moderner Krieg gegen den alten Feind? - Die Eisenbahnen im Deutsch-Französischen Krieg $1870 / 71$

Peter Jackson and Joseph Maiolo

Strategic intelligence, Counter-Intelligence and Alliance Diplomacy in Anglo-French relations before the Second World War

Ragna Boden

"Fortsetzung des Klassenkampfes mit anderen Mitteln «? Sowjetische Militärhilfepolitik gegenüber Entwicklungsländern am Beispiel der Republik Indonesien (1956-1965)

MiszeLLE

Thomas Freller

Korsarenjäger, General und Gesandter - Joseph Maria von Rechberg und Rothenlöwen

DOKUMENTATION

Stefan Martens und Walter Dreizner: Ein deutscher Soldat erlebt Friedrich-Rudolf Nagel die Befreiung von Paris im August 1944

\title{
NACHRICHTEN AUS DER FORSCHUNG
}

Annika Kropf und Holger Kozminski

Birte Löschenkohl, Tina Planck, Andreas Roessner und Patricia Wiegmann
Être en guerre - Erfahrung und Erinnerung. Der Zweite Weltkrieg in Europa. Internationales Kolloquium des Deutschen Historischen Instituts Paris und des Militärgeschichtlichen Forschungsamts, Potsdam in Zusammenarbeit mit den Deutschen Historischen Instituten London, Moskau, Rom und Warschau und dem Institut d'Histoire du Temps Présent Paris

545 toire Militaire" - Les relations franco-allemandes en matière d'armement au $X X^{\mathrm{e}}$ siècle: de la rivalité à la coopération 
Bernd Lemke

Thomas W. Probst

Markus Pöhlmann
»Krieg im Hinterland - Sicherheit im Innern?« Workshop am Militärgeschichtlichen Forschungsamt, Potsdam, 9. Dezember 2005

»Kriegsgräuel«. Tagung des Arbeitskreises Militärgeschichte (AKM), Mainz, 3. bis 5. November 2005

Die Rückkehr der Condottieri? Krieg und Militär im Spannungsfeld zwischen Verstaatlichung und Privatisierung. Die Entwicklung vom 16. Jahrhundert bis zur Gegenwart. Tagung, 12. bis 14. Mai 2006, Potsdam

\section{BUCHBESPRECHUNGEN}

Die Deutsche Kriegsgesellschaft 1939 bis 1945 . Erster Halbbd: Politisierung, Vernichtung, Überleben. Mit Beitr. von Rolf Blank [u.a.]. Im Auftr. des MGFA hrsg. von Jörg Echternkamp; Zweiter Halbbd: Ausbeutung, Deutungen, Ausgrenzung. Mit Beitr. von Bernhard Chiari [u. a.]. Im Auftr. des MGFA hrsg. von Jörg Echternkamp (= Das Deutsche Reich und der Zweite Weltkrieg, 9/1.2)

The Cambridge History of Warfare. Ed. by Geoff- Reiner Pommerin rey Parker

Aviezer Tucker, Our Knowledge of the Past. A Martin Meier Philosophy of Historiography

Kriegsniederlagen. Erfahrungen und Erinnerun- Jost Dülffer gen. Hrsg. von Horst Carl [u.a.]

Augenzeugen. Kriegsberichterstattung vom 18. Martin Moll zum 21. Jahrhundert. Hrsg. von Ute Daniel

Matthew Strickland, Robert Hardy, The Great Dirk Reitz Warbow. From Hastings to the Mary Rose bino 
Peter Engerisser, Von Kronach nach Nördlingen. Michael Kaiser Der Dreißigjährige Krieg in Franken, Schwaben und der Oberpfalz 1631-1635

Anja Rieck, Frankfurt am Main unter schwedi- Michael Peters scher Besatzung 1631-1635

Gerhard von Scharnhorst. Private und dienstliche Schriften. Bd 3: Lehrer, Artillerist, Wegbereiter Heinz Stübig (Preußen 1801-1804). Hrsg. von Johannes Kunisch in Verb. mit Michael Sikora

Andrew D. Lambert, Nelson. Britannia's god of war

Sven Externbrink

621

Englische Akten zur Geschichte des Krimkriegs. Michael Fröhlich 623 Bd 1: 20. November 1852 bis 10. Dezember 1853. Bearb. von Winfried Baumgart

Biographisches Handbuch des deutschen AuswärtiGünther Kronenbitter 625 gen Dienstes 1871-1945. Bd 2: G-K. Bearb.: Gerhard Keiper und Martin Kröger

Ewald Grothe, Zwischen Geschichte und Recht. Deutsche Verfassungsgeschichtsschreibung 1900-1970

Isabel V. Hull, Absolute Destruction. Military Culture and the Practices of War in Imperial Germany

Peter Arndt, Deutsche Sperrbrecher 1914-1945

Wolfgang Bühling

631

"Vorstellen könnt Ihr Euch den Krieg gar nicht, so schrecklich ist der«. Die Feldpost des Gladbecker Manfred 626 Schülers Franz Küster an seine Eltern (1915-1918). Hrsg. von Christine Schönebeck

Die Regierung Hitler. Bd 4: 1937. Bearb. von Fried- Michael Fröhlich 635 rich Hartmannsgruber

A World at Total War. Global Conflict and the Politics of Destruction, 1937-1945. Ed. by Roger Chickering, Stig Förster and Bernd Greiner

Markus Pöhlmann

John Keegan, Der Zweite Weltkrieg Rolf-Dieter Müller 639 Jörg Ganzenmüller, Das belagerte Leningrad 1941-1944

Enrico Syring 
Norbert Kunz, Die Krim unter deutscher Herr- Enrico Syring schaft (1941-1944)

Horst Steigleder, Die Kriegsmarine und der Ost- Jörg Hillmann feldzug

Bernhard R. Kroener, "Der starke Mann im Hei- Winfried Heinemann matkriegsgebiet « - Generaloberst Friedrich Fromm

Kurt Braatz, Gott oder ein Flugzeug. Leben und Heiner Möllers Sterben des Jagdfliegers Günther Lützow

Sönke Neitzel, Abgehört. Deutsche Generäle in Martin Kutz britischer Kriegsgefangenschaft 1942-1945

Welf Botho Elster, Die Grenzen des Gehorsams. Konrad Fuchs

Das Leben des Generalmajors Botho Henning

Elster in Briefen und Zeitzeugnissen

Lars-Broder Keil, Hans-Ulrich von Oertzen. Offi- Winfried Heinemann zier und Widerstandskämpfer

Kerstin von Lingen, Kesselrings letzte Schlacht. Horst Boog

Kriegsverbrecherprozesse, Vergangenheitspolitik und Wiederbewaffnung: Der Fall Kesselring

Geralf Gemser, Darf eine Schule diesen Namen Winfried Heinemann 660 tragen? Zur Vorbildwirkung des Wehrmachtsgenerals Erich Hoepner

Joachim Perels, Entsorgung der NS-Herrschaft? Ursula Hüllbüsch 662 Konfliktlinien im Umgang mit dem Hitler-Regime

Histoire de la coopération européenne dans l'arme- Florian Seiller 664 ment. Sous la dir. de Jean-Paul Hébert et Jean Hamiot

Christian Tuschhoff, Deutschland, Kernwaffen Klaus-Jürgen Bremm und die NATO 1949-1967 teidigungsplanung. Planung und Aufbau der Bundeswehr unter den Bedingungen einer massiven atomaren Vergeltungsstrategie 1952 bis 1960 
Israel und Deutschland. Dorniger Weg zur Part-

nerschaft. Die Botschafter berichten über vier Jahrzehnte diplomatische Beziehungen (1965-2005). Hrsg. von Asher Ben Natan und Niels Hansen

Asher Ben-Natan, Brücken bauen - aber nicht vergessen. Als erster Botschafter Israels in der Bundesrepublik (1965-1969)

Der Auswärtige Ausschuß des Deutschen Bundestages. Sitzungsprotokolle 1957-1961. Eingel. von Joachim Wintzer. Bearb. von Joachim Wintzer und Josef Boyer in Verb. mit Wolfgang Dierker. 2 Halbbde mit 1 CD-ROM

Documents Diplomatiques Français 1965, T. 1:

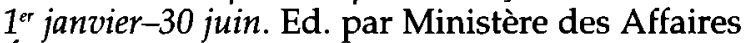
Étrangères

Documents Diplomatiques Français 1965, T. 2:

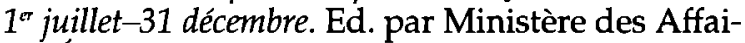
res Étrangères

Walter Jertz, Tornado. Technik - Taktik - Einsatz Heiner Möllers

684

Heeresmanöver der Bundeswehr. Hrsg.: Gerhard Helmut R. Brugmann in Zusammenarb. mit der Führungs- Hammerich akademie der Bundeswehr

Günther Glaser, "...auf die randere seite übergehen«. NVA-Angehörige in Krise und revoluRüdiger Wenzke tionärem Umbruch der DDR

Krieg an den Rändern. Von Sarajewo bis Kuito. Daniel Hildebrand 689 Hrsg. von Joachim Becker, Gerald Hödl und Peter Steyrer

Asymmetrische Kriegführung - ein neues Phänomen der Internationalen Politik? Hrsg. von Josef Martin Rink Schröfl und Thomas Pankratz

Deutsches Schiffahrtsarchiv 26/2003. Wissen- Jörg Hillmann 694 schaftliches Jahrbuch des Deutschen Schif688 fahrtsmuseums. Hrsg. von Uwe Schnall [u.a.] 


\section{Kollektivbiographie der Generäle}

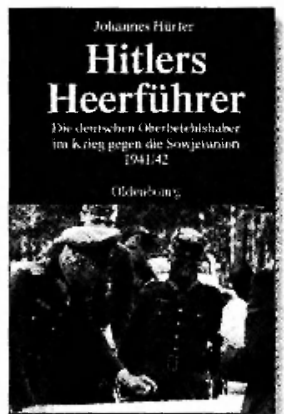

Johannes Hürter

Hitlers Heerführer

Die deutschen Oberbefehlshaber im Krieg

gegen die Sowjetunion 1941/42

2006 | VIII, 719 S. | 25 Abb. | 5 Karten

Ln. $\mid € 49,80$

ISBN 978-3-486-57982-6

Quellen und Darstellungen zur

Zeitgeschichte, Bd. 66

"Es ist zu wünschen, dass andere Historiker Hürters Beispiel folgen und genauso sauber gearbeitete Grundlagenstudien für die Zeit $a b$ 1942 und die übrigen Kriegsschauplätze, etwa für Italien oder den Balkan, vorlegen. Für die weiteren Studien des IfZ zur Geschichte der Wehrmacht hat Hürter mit der vorliegenden Arbeit den Maßstab vorgegeben." Alexander Brakel, in: H-Soz-u-Kult, September 2006

Wehrmacht in der NS-Diktatur. Ein Projekt des Instituts für Zeitgeschichte

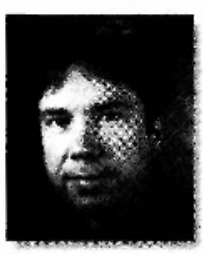

Dr. Johannes Hürter ist wissenschaftlicher Mitarbeiter am Institut für Zeitgeschichte München-Berlin, Redakteur der Vierteljahrshefte für Zeitgeschichte und Dozent an der Universität der Bundeswehr München-Neubiberg.

Bestellungen über den Buchhandel oder direkt: o89/45051-333, E-Mail: verkauf@oldenbourg.de oldenbourg.de 